\title{
The 2019 Nobel Prize honors fundamental discoveries in hypoxia response
}

\author{
Javid Moslehi' ${ }^{1,2}$ and W. Kimryn Rathmell2,3 \\ 'Division of Cardiovascular Medicine, Cardio-Oncology Program, ${ }^{2}$ Department of Medicine, Vanderbilt-Ingram Cancer Center, and ${ }^{3}$ Division of Hematology and Oncology, Vanderbilt University Medical Center \\ (VUMC), Nashville, Tennessee, USA.
}

$\mathbf{T}$ 2019 Nobel Prize for Physiology or Medicine was awarded to Professors Sir Peter J. Ratcliffe (University of Oxford), Gregg L. Semenza (Johns Hopkins University), and William G. Kaelin Jr. (DanaFarber Cancer Institute) for their discoveries of a fundamental aspect of cellular physiology, the cellular sensing of oxygen levels and regulation of physiologic hypoxia. Each of these physician-scientists was intrigued by a different clinically relevant observation and utilized very basic biochemical tools to address their questions. These separate lines of investigation converged, delineating a central cellular pathway with far-ranging implications for human physiology, disease states, and medicine. The story of how they uncovered hypoxia response begins with a deep interest in basic human biology.

\section{Uncovering a hypoxia-inducible pathway}

Semenza, a pediatric geneticist, was studying the triggers for the production of erythropoietin, a hematopoietic growth factor produced by the liver and kidney that promotes the generation of red blood cells. His group identified a sequence-specific binding site (termed hypoxia-response element [HRE]) for a transcription factor in the $3^{\prime}$ flanking region of the human erythropoietin gene (EPO). He biochemically purified the factor, which he called hypoxiainducible factor 1 (HIF-1) (1). HIF-1 is a heterodimer composed of HIF-1 $\alpha$, a protein expressed in an oxygen-regulated manner, and a constitutively expressed factor, HIF$1 \beta$. This dimer formed a potent transcription factor complex, and Semenza showed that in addition to erythropoietin, HIF-1 induces a number of other genes that are critical for cellular and systemic response to hypoxia, and maintenance of cellular homeostasis. Among these was vascular endothelial growth factor (VEGF), which plays important roles in angiogenesis (2).

\section{A cancer connection}

Simultaneously, Kaelin was studying von Hippel-Lindau (VHL) disease, a rare genetic syndrome in which mutations in the affected gene $(V H L)$ lead to specific cancer types, such as renal cell carcinoma (RCC) and cerebellar hemangioblastomas. Trained as an internist (he was chief resident of the Johns Hopkins Osler service) and oncologist, he was intrigued by VHL syndrome because the associated tumors were always highly vascular in nature, with affected patients occasionally having secondary polycythemia. Pivotal biochemical studies showed that the protein product of $V H L$ (pVHL) forms a complex that plays an important role in the ubiquitination and subsequent degradation of specific proteins. Importantly, the proper assembly of the VHL complex was critical for regulation of hypoxia-inducible genes (3). X-ray crystallography of the VHL complex suggested that pVHL serves as an E3 ubiquitin ligase, conferring specificity to the ubiquitination and subsequent degradation of one or more proteins (4). The quest to find the protein was on.

\section{Putting the pieces together}

A crucial observation connecting these lines of research came from the Ratcliffe group. Ratcliffe, a nephrologist,

Conflict of interest: JM has served on an advisory boards for Pfizer, Novartis, Bristol-Myers Squibb, Audentes Therapeutics, Nektar, and AstraZeneca. WKR serves on the Board of Scientific Advisors for the National Cancer Institute, and on the scientific advisory board for Aravive. WKR serves as the lead investigator for clinical trials at VUMC supported by Pfizer, Novartis, Bristol-Myers Squibb, Peloton, Calithera, and Roche.

Copyright: () 2020, American Society for Clinical Investigation.

Reference information: / Clin Invest. 2020;130(1):4-6. https://doi.org/10.1172/JCI134813.

was intrigued by how kidneys serve as oxygen-sensing organs and thus regulate the production of erythropoietin systemically. In early experiments, his group showed that multiple cell types are capable of sensing hypoxia and driving EPO transcription via the HRE that Semenza had identified (5). This suggested that HIF possibly serves as a universal response mechanism to hypoxia. Importantly, in 1999, the Ratcliffe team showed that pVHL regulates HIF- $1 \alpha$ and most likely acts as its E3 ubiquitin ligase (6).

\section{Regulating the VHL-HIF interaction}

The mechanisms underpinning HIF-1 $\alpha$ stabilization and activation of a massive transcriptional program of hypoxia response were elegantly delineated by the Kaelin, Semenza, and Ratcliffe groups, revealing a beautifully simple model. During normoxia, when oxygen is ample, specific proline residues on HIF-1 $\alpha$ undergo hydroxylation. This covalently modified form of HIF-1 $\alpha$ is recognized in a contact-dependent manner by pVHL, marking HIF- $1 \alpha$ for ubiquitination and proteasome-mediated destruction. During hypoxia, HIF-1 $\alpha$ is not hydroxylated and escapes tagging for degradation $(7,8)$. This suggested that regulation of HIF is posttranscriptional: HIF-1 $\alpha$ is transcribed, translated, and quickly degraded, unless oxygen becomes limiting. Notably, a second homologous protein, HIF$2 \alpha$, was identified, featuring identical hydroxylation-dependent regulation via VHL. HIF-1/2 $\alpha$, when stabilized, rapidly heterodimerizes with HIF-1 $\beta$, transcribing hundreds of genes that are critical for the cellular response to hypoxia. The genes involved in this tightly controlled response pathway include notable heavyweights such as VEGF, erythropoietin, mediators of glycolysis and other metabolic pathways, and genes involved in cell 


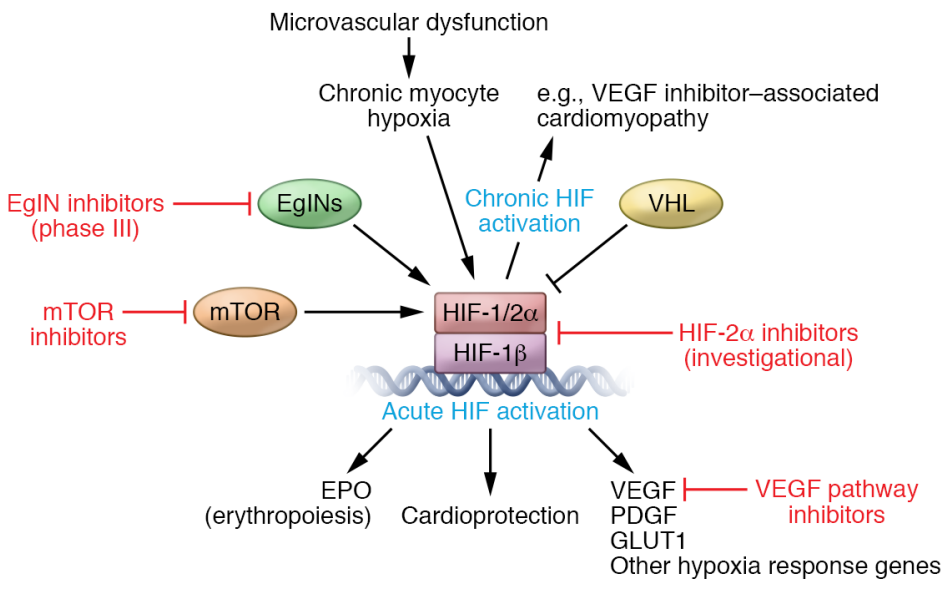

Figure 1. The discovery of the VHL/EgIN/HIF pathway as a central mediator of hypoxia signaling has paved the way for introduction of drugs that modulate the pathway for the treatment of human disease. Because the pathway is so central to so many pathological conditions, pharmacologics are being used for the treatment of cancer, as well as being tested for other conditions, such as anemia and cardiovascular and pulmonary diseases. Therapeutics targeting this pathway include VEGF pathway inhibitors (bevacizumab, sorafenib, sunitinib, pazopanib, axitinib, cabozantinib, and lenvatinib), mTOR inhibitors (temsirolimus and everolimus), EgIN inhibitors in phase III trials (daprodustat, molidustat, roxadustat, and vadadustat), and investigational HIF-2 $\alpha$ inhibitors (PT2385, PT2977, and RNAi [phase I]).

survival and homeostasis - and new target genes continue to be uncovered.

Knowing the importance of hydroxylation in the recognition of HIF-1 $\alpha$ by pVHL, the hunt to identify the responsible enzymes began. Prolyl hydroxylation had been well defined in the setting of stabilization of procollagen chains. There were striking similarities; both reactions required oxygen, iron, and ascorbate, and were inhibited by cobalt and by 2-oxoglutarate analogs, suggesting that the HIF prolyl hydroxylase was a member of the 2-oxoglutrate-dependent dioxygenase enzyme family. A group of enzymes were identified as the key oxygen sensors based on the discovery of the egl9 protein in Caenorhabditis elegans: the EglN (Egl nine homolog), or PHD (prolyl hydroxylase domain), proteins. These three orthologs catalyze hydroxylation of human HIF-1/2 $\alpha$ (9-11). EglN1 (also called prolyl hydroxylase domain-containing protein-2 [PHD2]) was shown to be the critical hydroxylase for HIF- $\alpha$ in vivo (12). The requirement for 2-oxoglutate for the reaction provided an additional link between hypoxia and metabolism. EglNs' unusual low oxygen affinity suggested that these molecules were indeed oxygen sensors, an observation that was confirmed genetically. In murine models in which EglN1 is temporally genetically deleted, mice display hypervascular features and polycythemia (12). The phenotype of these mice mimicked responses to high altitude. Further, inactivating mutations in EglN1 or activating mutations in HIF- $2 \alpha$ have been shown to cause familial erythrocytosis (13, 14). Even more intriguing, single nucleotide polymorphisms (SNPs) or missense mutations in EglN1 allow for high-altitude adaptation in populations living in high altitudes (e.g., Tibet) (15).

\section{Hypoxia in the patient setting}

Let's put the discoveries of hypoxia response in context with a clinical case: A middle-aged woman was admitted to the hospital with pathologic fracture of the femur as a result of metastatic clear cell RCC. Past medical history was significant for coronary artery disease, and she had mild cardiomyopathy. A smoker, she had chronic kidney disease and anemia. Hypoxia signaling played a central role in nearly every feature of her disease process, providing for an outstanding teaching opportunity with the house staff team.

This patient's cancer is likely driven by inactivation of the VHL gene. She had been treated for 11 months with a VEGFR inhibitor, with good disease control. The model proposed by which VHL loss resulted in stabilization of HIF factors and transcriptional activation of hypoxia- associated genes immediately explained why VHL syndrome-associated cancers were hypervascular. Somatic mutations in $V H L$ are tightly linked to sporadic clear cell RCC. Not surprisingly, clear cell RCC is one of the select groups of cancers for which VEGF pathway-directed therapy (Figure 1) has been proven to induce substantial responses in tumor volume and an increase in overall survival (16). This discovery can be directly linked to the emergence of a class of drugs targeting VEGF signaling that have become a staple of the cancer targeted therapy armamentarium.

Critical for our patient, the physiologic and chronic response to hypoxia resulting from coronary artery disease, damaged lung epithelium, and recurrently injured kidneys plays important roles in tissue remodeling and disease states. VEGFR inhibition may have potentially contributed directly to microvascular dysfunction, myocardial hypoxia, and (ironically) stabilization of HIF in myocytes and subsequent myocardial dysfunction (17). Hypoxia signaling alters the metabolic properties of these tissues, shifts the balance of reactive oxygen species, and accumulates free radical-induced damage. Hypoxia-mediated effects can impact any tissue in which delivery of oxygen and nutrients is critical, wherein hypoxia signaling underlies serious nonmalignant and malignant conditions.

The bone marrow and kidneys are worthy of special consideration in this case. Hypoxic signaling acts as a rheostat that maintains the necessary growth signals for stem cells. The bone marrow niche is where blood stem cells reside, and perturbations - such as infiltration with cancer cells, fractures and disrupted blood flow, and radiation exposure - have the potential to damage this critical space. Similarly, cells with exquisite sensory capacity, predominately localized in the kidney, utilize HIF signaling to maintain erythropoietin production that stimulates erythroid precursor differentiation in that marrow niche. End organ damage at either site can significantly impact a patient's ability to keep up with demand for red cell production.

\section{Conclusions}

The discoveries by Semenza, Kaelin, and Ratcliff began with a keen focus on fundamental aspects of human physiology: 
signaling that alters red blood cell production and ultimately oxygen carrying capacity, and the vasculature that delivers that essential cargo. In the realm of cancer, while VHL-associated tumors and RCC display a remarkable pseudohypoxic state and vascular dependence due to constitutive stabilization of HIF- $2 \alpha$, in many other tumor types one or both HIF factors are stabilized or upregulated, which can frequently be a potent biomarker of aggressive tumors. Importantly, small molecules that target HIF proteins are emerging as cancer therapeutics (18).

As a result of these seminal discoveries, therapies that dampen hypoxic response have also been applied or tested in other settings, including retinal diseases, pulmonary hypertension, cardiovascular disease, renovascular disease, and immune regulation. Conversely, therapies are being developed that apply a precision approach to selectively stabilize HIF, inhibiting the EglN/HIF axis as a treatment for patients with chronic anemia and chronic kidney disease (19), bringing the original observations full circle.

The three physician-scientists honored with the 2019 Nobel Prize in Physiology or Medicine highlight the tight coupling between clinical observations and fundamental aspects of human biology, inspire future physicians to apply that level of attention to clinical medicine, and pave the way for innovative therapeutic advances through their unwavering pursuit of basic research into the mechanisms by which cells sense and respond to oxygen.

\section{Acknowledgments}

JM is supported by NIH R56 HL141466 and R01 HL141466. WKR is supported by R01 CA198482 and R01 CA203012.

Address correspondence to: W. Kimryn Rathmell, Division of Hematology and Oncology, Vanderbilt University Medical Center, 2220 Pierce Avenue, Preston Research Building, Suite 798C, Nashville, Tennessee 37232, USA. Phone: 615.875.9731; Email: Kimryn.Rathmell@ VUMC.org

1. Wang GL, Semenza GL. Purification and characterization of hypoxia-inducible factor 1. J Biol Chem. 1995;270(3):1230-1237.

2. Forsythe JA, et al. Activation of vascular endothelial growth factor gene transcription by hypoxia-inducible factor 1. Mol Cell Biol. 1996;16(9):4604-4613.

3. Lonergan KM, et al. Regulation of hypoxiainducible mRNAs by the von Hippel-Lindau tumor suppressor protein requires binding to complexes containing elongins $\mathrm{B} / \mathrm{C}$ and $\mathrm{Cul} 2$. Mol Cell Biol. 1998;18(2):732-741.

4. Stebbins CE, Kaelin WG, Pavletich NP. Structure of the VHL-ElonginC-ElonginB complex: implications for VHL tumor suppressor function. Science. 1999;284(5413):455-461.

5. Maxwell PH, Pugh CW, Ratcliffe PJ. Inducible operation of the erythropoietin $3^{\prime}$ enhancer in multiple cell lines: evidence for a widespread oxygen-sensing mechanism. Proc Natl Acad Sci U S A. 1993;90(6):2423-2427.

6. Maxwell PH, et al. The tumour suppressor protein VHL targets hypoxia-inducible factors for oxygen-dependent proteolysis. Nature. 1999;399(6733):271-275.

7. Jaakkola P, et al. Targeting of HIF- $\alpha$ to the von Hippel-Lindau ubiquitylation complex by $\mathrm{O}_{2}$-regulated prolyl hydroxylation. Science. 2001;292(5516):468-472.
8. Ivan M, et al. HIFalpha targeted for VHLmediated destruction by proline hydroxylation: implications for $\mathrm{O}_{2}$ sensing. Science. 2001;292(5516):464-468.

9. Epstein AC, et al. C. elegans EGL-9 and mammalian homologs define a family of dioxygenases that regulate HIF by prolyl hydroxylation. Cell. 2001;107(1):43-54.

10. Bruick RK, McKnight SL. A conserved family of prolyl-4-hydroxylases that modify HIF. Science. 2001;294(5545):1337-1340.

11. Ivan $\mathrm{M}$, et al. Biochemical purification and pharmacological inhibition of a mammalian prolyl hydroxylase acting on hypoxiainducible factor. Proc Natl Acad Sci U S A. 2002;99(21):13459-13464

12. Minamishima YA, Moslehi J, Bardeesy N, Cullen D, Bronson RT, Kaelin WG. Somatic inactivation of the PHD2 prolyl hydroxylase causes polycythemia and congestive heart failure. Blood. 2008;111(6):3236-3244.

13. Percy MJ, et al. A family with erythrocytosis establishes a role for prolyl hydroxylase domain protein 2 in oxygen homeostasis. Proc Natl Acad Sci US A. 2006;103(3):654-659.

14. Percy MJ, et al. A gain-of-function mutation in the HIF2A gene in familial erythrocytosis. $N$ Engl JMed. 2008;358(2):162-168.

15. Simonson TS, et al. Genetic evidence for high-altitude adaptation in Tibet. Science. 2010;329(5987):72-75

16. Rini BI. Targeted therapy for patients with renal-cell carcinoma. Lancet Oncol. 2011;12(12):1085-1087.

17. Moslehi JJ. Cardiovascular toxic effects of targeted cancer therapies. $N$ Engl J Med. 2016;375(15):1457-1467.

18. Courtney KD, et al. Phase I dose-escalation trial of PT2385, a first-in-class hypoxia-inducible factor- $2 \alpha$ antagonist in patients with previously treated advanced clear cell renal cell carcinoma. JClin Oncol. 2018;36(9):867-874.

19. Chen N, et al. Roxadustat for anemia in patients with kidney disease not receiving dialysis. N Engl J Med. 2019;381(11):1001-1010. 